\section{Was macht} „nano" im Müll?

\section{In Kürze}

- Immer mehr Produkte enthalten Nanomaterialien. Diese landen früher oder später auch im Abfall. Wie sie sich dort verhalten, ist ungewiss.

- Es liegen kaum Informationen über die in Produkten eingesetzte Nanomaterialien und deren Mengen vor.

- Das stellt die österreichische Abfallwirtschaft und Gesetzgebung vor Herausforderungen.

- Lösungsansätze sind unter anderem ein einheitliches Register zur Angabe von Nanomengen in Produkten, eine verbesserte Kennzeichnung sowie die Überprüfung der Arbeitsplatzsicherheit im Abfallbereich.

\section{Worum geht es?}

Künstlich hergestellte Nanomaterialien werden seit vielen Jahren in Alltagsprodukten verarbeitet und landen damit auch im Müll oder im Abwasser. Aktuelle Beispiele sind NanoTitandioxid in Sonnencremes oder Kohlenstoff-Nanoröhrchen in Tennisschlägern. Weltweit und in Österreich nimmt die Anzahl von Nanoprodukten am Markt zu.

Häufig weisen Nanopartikel aufgrund ihrer geringen Größe von wenigen Millionstel Millimetern besondere Eigenschaften auf. Das macht sie für den Einsatz in bestimmten Produkten besonders interessant. Sie reagieren allerdings chemisch mitunter anders als das gleiche Material in größerer Dimension. Deswegen können Nanopartikel unter Umständen negative Auswirkungen auf die Umwelt haben, beispielsweise im Grundwasser oder im Boden. Nanomaterialien könnten bei entsprechender Menge auch ein gesundheitliches Risiko darstellen, wenn sie etwa von Angestellten in Abfallbehandlungsanlagen direkt eingeatmet oder langfristig indirekt über die Nahrungskette aufgenommen werden.
Das aktuelle Abfallwirtschaftsrecht zeigt aber keine nanospezifischen Regulierungen. Nanomaterialien sind zwar theoretisch in bestehende Grenzwerte integriert. Aufgrund inrer einzigartigen Eigenschaften und ihrer höheren Reaktivität bei geringer Masse sind Grenzwerte für Nanomaterialien jedoch häufig viel zu hoch angesetzt, um wirksam zu werden.

Aber in welchen Alltagsprodukten sind Nanomaterialien enthalten? Was passiert mit innen wenn sie entsorgt werden? Und wo landen sie letztendlich in der Abfallverwertung?

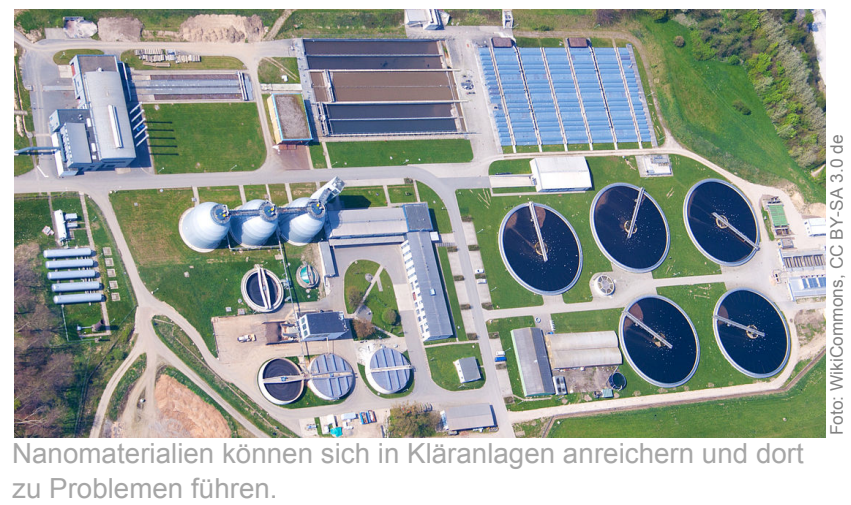

Es ist schwierig festzustellen, ob Produkte künstlich hergestellte Nanomaterialien enthalten oder nicht. Lediglich für Kosmetika gibt es seit 2013 innerhalb der Europäischen Union eine offizielle Kennzeichnungspflicht. Für alle anderen Produktgruppen gilt: Solange der Inhaltsstoff als nicht bedenklich eingestuft wird, steht es den Herstellern frei, die Verwendung von Nanomaterialien anzugeben. Der Nachweis von Nanomaterialien ist freilich derzeit technisch noch aufwändig.

Es gibt daher keine umfassenden Informationen, welche Produkte Nanomaterialien enthalten. Auch genaue Mengenangaben von spezifischen Nanomaterialien für Österreich sind nicht verfügbar - bestenfalls gibt es Schätzungen und Hochrechnungen für einzelne Materialien. Die Abfallwirtschaft kann sich daher nur schwierig auf nanohaltigen Abfall einstellen. Dies wäre aber im Sinne des Vorsorgeprinzips dringend notwendig, weil Nanomaterialien die technische Ausstattung von Abfallbehandlungsanlagen vor neue Herausforderungen stellen könnten.

\section{Eckdaten}

Projekttitel: Projektteam:

NanoMia - NanoMüll im Abfall Gazsó, A., Fuchs, D. im Rahmen einer Kooperation (Leitung: ABF, BOKU Wien)

Laufzeit: 04/2014 - 03/2015

Auftraggeber: BMLFUW, BMVIT Website: oeaw.ac.at/ita/projekte/ nanomia/ueberblick 


\section{Wesentliche Ergebnisse}

Die Nanoproduktdatenbank des ITA bietet einen Überblick über Produkte am österreichischen Markt, die Nanomaterialien enthalten. Abgesehen von Kosmetika, die verpflichtend gekennzeichnet werden müssen, beruhen die Einträge jedoch auf freiwilligen Angaben der Hersteller (z.B. zu Werbezwecken) oder weil bekannt ist, dass nanotechnologische Verfahren im Herstellungsprozess angewandt wurden (z.B. Sol-GelVerfahren). Damit wird nur ein Teil der Nanoprodukte, die in Österreich erhältlich sind, erfasst.

Aus den über 490 Einträgen wurden sechs Alltagsprodukte ausgewählt, die je ein charakteristisches Nanomaterial enthalten und unterschiedlich genutzt werden. Untersucht wurde Nano-Titandioxid in Sonnencremes, Nano-Silber in Innenund Außenwandfarben, Kohlenstoff-Nanoröhrchen in Sportequipment, Nano-Siliziumdioxid in Autozubehör wie Lacken, Nano-Silber in Einweg-Putztüchern sowie Quantenpunkte in modernen Fernsehgeräten.

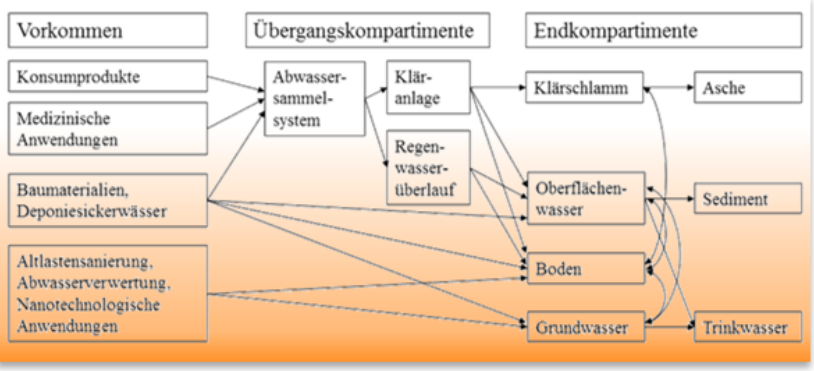

Wo fallen Nanomaterialien an (Vorkommen)? Über welche Abfallund Abwasserbehandlungssysteme (Übergangskompartimente) werden sie in die Umwelt ausgetragen (Endkompartimente)?

Der Abfallbehandlungsweg ist für jedes dieser Produkte unterschiedlich. Stofffluss-Analysen illustrieren, welche Wege Nanomaterialien im Abfallsystem nehmen.

So können diese Wege klar dargestellt und jene Punkte im Abfallsystem identifiziert werden, an denen Nanopartikel in die Umwelt (Wasser, Boden oder Luft) ausgetragen werden könnten. Dabei geht es vorrangig darum aufzuzeigen, wo genau Nanomaterialien sich im österreichischen Abfallsystem wieder finden könnten, sobald sie in Massenprodukten eingesetzt und damit auch entsorgt werden.

Da es im Moment häufig keine verlässlichen Daten dazu gibt, sind akkurate Berechnungen von Mengenangaben jedoch kaum möglich. Für Quantenpunkte konnte beispielsweise keine Stoffflussanalyse erstellt werden, da keine Angaben oder Schätzungen über eingesetzte Mengen in Elektronikgeräten vorliegen. Fehlende Mengenangaben sind aber problematisch, weil diese zur Abschätzung von Umweltauswirkungen dringend notwendig sind.

\section{Was tun?}

Die Risiko-Regulierung von Nanomaterialien in Abfallströmen stellt Politik und Gesellschaft vor neue Herausforderungen. Dazu müssen sowohl wissenschaftliche, als auch abfalltechnische und gesetzliche Rahmenbedingungen angepasst werden.

- Es braucht mehr Daten zu Nanomaterialien in Produkten. Dazu ist eine Erhebung von Mengenangaben in Produkten oder Produktionsmengen notwendig - etwa durch ein einheitliches EU-weites Register oder die Anpassung der Europäischen Chemikalien-Verordnung $\mathrm{REACH}$.

- Arbeitsplätze im Abfallbereich müssen sicher sein: Hier können potenziell Nanopartikel in Bearbeitungsprozessen wieder freigesetzt werden.

- Die Nanoprodukt-Kennzeichnung sollte verpflichtend sein und mehr Informationen beinhalten.

- Um ein adäquates Recycling und die sachgemäße Entsorgung zu gewährleisten, sind verpflichtende Rücknahmeverfahren sinnvoll. Voraussetzung dafür ist allerdings die Verfügbarkeit von ausreichend Information zu Nano-Produkten. Ansätze dazu sind etwa die erweiterte Produzentenverantwortung der EU-Abfallrahmenrichtlinie 2008 oder die Environmental Product Declaration (EPD).

- Nanospezifische Analytik und Standardverfahren zur Identifikation von künstlich hergestellten Nanomaterialien in gemischten Abfallmengen müssen weiterentwickelt werden. Notwendig wären standardisierte Nachweisverfahren für bestimmte Nanopartikel und Methoden zur Analyse der Veränderung bestimmter Eigenschaften von Nanomaterialien über den Lebenszyklus.

- Bestehende Technologien und Maßnahmen im Abfallbereich müssen auf ihre Eignung im Umgang mit Nanomaterialien hin überprüft werden.

\section{Zum Weiterlesen}

NanoTrust-Dossier Nr. 043.(2015) Umweltrelevante Aspekte von Nanomaterialien am Ende der Nutzungsphase - Teil I: Abwässer und Klärschlamm epub.oeaw.ac.at/ita/nanotrust-dossiers/dossier043.pdf

\section{Kontakt}

\section{André Gazsó}

E-mail: tamail@oeaw.ac.at

Telefon: +43(1)51581-6582

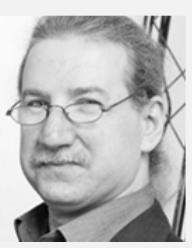

IMPRESSUM: Medieninhaber: Österreichische Akademie der Wissenschaften; Juristische Person öffentlichen Rechts (BGBI. 569/1921 i.d.F. BGBI. I 130/2003); Dr. Ignaz Seipel-Platz 2, A-1010 Wien; Herausgeber: Institut für Technikfolgen-Abschätzung (ITA); Strohgasse 45/5, A-1030 Wien; www oeaw. ac.at/ita | Erscheinungsweise: Die ITA-Dossiers erscheinen unregelmäßig und dienen der Veröffentlichung der Forschungsergebnisse des ITA. Die Dossiers werden in kleiner Auflage gedruckt und open access über das Portal „epub.oeaw“ zur Verfügung gestellt: epub.oeaw.ac.at/ita/ita-dossiers | ISSN: 2306-1960 @) (i) Dieses Werk ist lizenziert unter einer Creative Commons Namensnennung 4.0 International Lizenz: creativecommons org/licenses/by/4 0/deed:de ITA-Dossier Nr. 18, Dezember 2015, epub. oeaw. ac. at/ita/ita-dossiers/ita-dossier018 dodf | Hauptautorin: D. Fuchs 